\title{
HDAC1 and HDAC2 Double Knockout Triggers Cell Apoptosis in Advanced Thyroid Cancer
}

Ching-Ling Lin ${ }^{1,2}{ }^{\oplus}$, Ming-Lin Tsai ${ }^{3}$, Chun-Yu Lin ${ }^{4,5} \oplus^{\circ}$, Kai-Wen Hsu ${ }^{6,7}$, Wen-Shyang Hsieh ${ }^{8}$, Wei-Ming Chi ${ }^{9}$, Li-Chi Huang ${ }^{1,2, *}$ and Chia-Hwa Lee ${ }^{8,10,11,12, *}$

1 Department of Endocrinology and Metabolism, Cathay General Hospital, Taipei 10630, Taiwan; work5halfday@cgh.org.tw

2 Department of Internal Medicine, School of Medicine, College of Medicine, Taipei Medical University, Taipei 11031, Taiwan

3 Department of General Surgery, Cathay General Hospital, Taipei 10630, Taiwan; hyperexias@yahoo.com.tw

4 Institute of Bioinformatics and Systems Biology, National Chiao Tung University, Hsinchu 30068, Taiwan; chunyulin.bi99g@g2.nctu.edu.tw

5 Bioinformatics Center, Institute for Chemical Research, Kyoto University, Kyoto 611-0011, Japan

6 Institute of New Drug Development, China Medical University, Taichung 40402, Taiwan; kwhsu@mail.cmu.edu.tw

7 Research Center for Tumor Medical Science, China Medical University, Taichung 40402, Taiwan

8 Department of Medical Laboratory, Taipei Medical University—Shuang Ho Hospital, New Taipei City 23561, Taiwan; 12638@s.tmu.edu.tw

9 Department of Clinical Pathology, Taipei Medical University-Shuang Ho Hospital, New Taipei City 23561, Taiwan; rc4202@tmu.edu.tw

10 School of Medical Laboratory Science and Biotechnology, College of Medical Science and Technology, Taipei Medical University, Taipei 11031, Taiwan

11 Ph.D. Program in Medicine Biotechnology, College of Medicine, Taipei Medical University, Taipei 11031, Taiwan

12 TMU Research Center of Cancer Translational Medicine, Taipei 11031, Taiwan

* $\quad$ Correspondence: likih@seed.net.tw (L.-C.H.); chlee@tmu.edu.tw (C.-H.L.); Fax: +886-2-2739-5133 (C.-H.L.)

Received: 6 December 2018; Accepted: 17 January 2019; Published: 21 January 2019

check for updates

\begin{abstract}
Anaplastic thyroid carcinoma (ATC) and squamous thyroid carcinoma (STC) are both rare and advanced thyroid malignancies with a very poor prognosis and an average median survival time of 5 months and less than $20 \%$ of affected patients are alive 1 year after diagnosis. The clinical management of both ATC and STC is very similar because they are not particularly responsive to radiotherapy and chemotherapy. This inspired us to explore a novel and effective clinically approved therapy for ATC treatment. Histone deacetylase inhibitor (HDACi) drugs are recently FDA-approved drug for malignancies, especially for blood cell cancers. Therefore, we investigated whether an HDACi drug acts as an effective anticancer drug for advanced thyroid cancers. Cell viability analysis of panobinostat treatment demonstrated a significant IC50 of $0.075 \mu \mathrm{M}$ on SW579 STC cells. In addition, panobinostat exposure activated histone acetylation and triggered cell death mainly through cell cycle arrest and apoptosis-related protein activation. Using CRISPR/Cas9 to knock out HDAC1 and HDAC2 genes in SW579 cells, we observed that the histone acetylation level and cell cycle arrest were enhanced without any impact on cell growth. Furthermore, HDAC1 and $H D A C 2$ double knockout $(\mathrm{KO})$ cells showed dramatic cell apoptosis activation compared to HDAC1 and $H D A C 2$ individual $\mathrm{KO}$ cells. This suggests expressional and biofunctional compensation between HDAC1 and HDAC2 on SW579 cells. This study provides strong evidence that panobinostat can potentially be used in the clinic of advanced thyroid cancer patients.
\end{abstract}

Keywords: anaplastic thyroid carcinoma; squamous thyroid carcinoma; histone deacetylase inhibitor; CRISPR/Cas9; knockout 


\section{Introduction}

According to the National Cancer Institute, there are over 56,000 new cases of thyroid cancer in the USA each year and the majority of those diagnosed are papillary thyroid cancer, which is the most common type of thyroid cancer. Among all thyroid cancers, squamous thyroid carcinoma (STC) and anaplastic thyroid carcinoma (ATC) are rare neoplasms and have been reported in less than $1-2 \%$ of all thyroid cases [1,2]. STC and ATC are typically diagnosed as advanced thyroid malignancies with infiltration in adjacent organs, including the larynx, esophagus and trachea with a poor prognosis, where the median overall survival time is 6 months in clinical patients [3]. Due to the extremely aggressive behavior of ATC, the American Joint Committee on Cancer (AJCC) defines all of its stages as stage IV [4]. In the clinical management of advanced thyroid malignancy, surgical treatment is not always possible and the available radiotherapy/chemotherapy for thyroid cancer is always relatively ineffective [5]. Therefore, it is necessary to research highly efficient and low toxicity drugs to treat advanced thyroid cancers.

Histone modification is a covalent posttranslational modification (PTM) to histone proteins, which includes methylation, phosphorylation, acetylation, ubiquitylation and sumoylation. Among these PTMs, the regulation of histone acetylation and methylation have been well investigated in tumorigenesis, which manipulates the activation/inactivation of both oncogenic and tumor suppressive gene transcription. Histone deacetylases (HDACs) are epigenetic regulators that coordinate histone proteins, chromatin conformation and protein-DNA interactions. In many respects, histone deacetylase inhibitors (HDACis) represent prototypical epigenetic agents that act by modifying gene expression to restore the normal differentiation or death programs of transformed cells. To date, the USA FDA has approved four HDACis, including vorinostat (SAHA, 2006), romidepsin (FK228, 2009), belinostat (PXD101, 2014) and panobinostat (LBH-589, 2015), which are widely used as anticancer drugs, especially for refractory cutaneous and peripheral $\mathrm{T}$ cell lymphoma and multiple melanoma. Increasing evidence suggests that epigenetic alternations (DNA methylation, acetylation and chromatin modification) play key roles in thyroid cancer development, as well as cancer cell growth and differentiation [6-10]. Previous studies have shown that epigenetic modification causes gene silencing, which substantially decreases the responsiveness of thyroid tumors to radioiodine therapy [11-13]. Furthermore, epigenetic gene modifications also contribute to the dysregulation of both cell proliferation and cancer development, including apoptosis and cancer cell migration and invasion abilities.

In the current study, we investigated whether HDACi drugs, including panobinostat, belinostat, vorinostat and valproic acid, possess anticancer activity against advanced thyroid cancer, especially for SW579 STC cells. Further epigenetic alterations involved in cell cycle arrest and apoptosis were also investigated. Using our developed techniques, which combine lentiviral transfection and noninvasive apoptosis detection sensor (NIADS) analysis [14], we were able to quantitatively and kinetically analyze apoptotic cell death in HDACi-treated SW579 cells. HDAC1 and HDAC2 are two of the most investigated proteins in balancing histone acetylation and chromatin remodeling. By knocking out HDAC1 and HDAC2 using clustered regularly interspaced short palindromic repeats (CRISPR/Cas9), we investigated the impact of HDAC loss and how acetylation is regulated in thyroid cancer cells and observed the downstream signaling pathways and cell cycle regulation. In summary, this study could help us establish the basis of the pharmacological effects on HDAC regulation in future HDACi drug design targeting human advanced thyroid cancer.

\section{Results}

\subsection{FDA-Approved HDACi Induced SW579 Cell Apoptosis}

Squamous-cell thyroid carcinoma (STC) cells were treated with different concentrations of four FDA-approved clinical HDACi drugs (panobinostat, belinostat, vorinostat and valproic acid) for the cell viability assay (Figure 1A). After HDACi drug exposure for $24 \mathrm{~h}$, cells treated with panobinostat had a relatively low cell viability at $0.1 \mu \mathrm{M}(50 \%)$ compared to belinostat $(73.1 \%)$, vorinostat $(93.3 \%)$ 
and valproic acid (99\%) treatments. In addition, by investigating different doses of panobinostat treatment, SW579 cells had a lower cell viability at 1 and $10 \mu \mathrm{M}(29.4 \%$ and $28.2 \%$, respectively), whereas belinostat and vorinostat reached the maximum anticancer effect at $10 \mu \mathrm{M}(30 \%$ and $47.5 \%$, respectively) treatments. After analysis of the IC50, we observed that the IC50 of panobinostat is $0.075 \mu \mathrm{M}$, whereas belinostat and vorinostat have IC50 drug concentrations of 2.129 and $9.141 \mu \mathrm{M}$, respectively. Using a live/dead cell assay (Figure 1B), it is clear that exposure to $1 \mu \mathrm{M}$ panobinostat and belinostat for $24 \mathrm{~h}$ caused significant cell death effects (red), with $142 \%$ and $120 \%$ dead/live cell ratios $(p<0.01)$, respectively, whereas vorinostat and valproic acid had relatively minor effects on cell death in SW579 cells. These cell viability results clearly indicate that panobinostat is one of the most effective anticancer drugs among the HDACi drugs on squamous-cell thyroid carcinoma of advanced thyroid cancer.

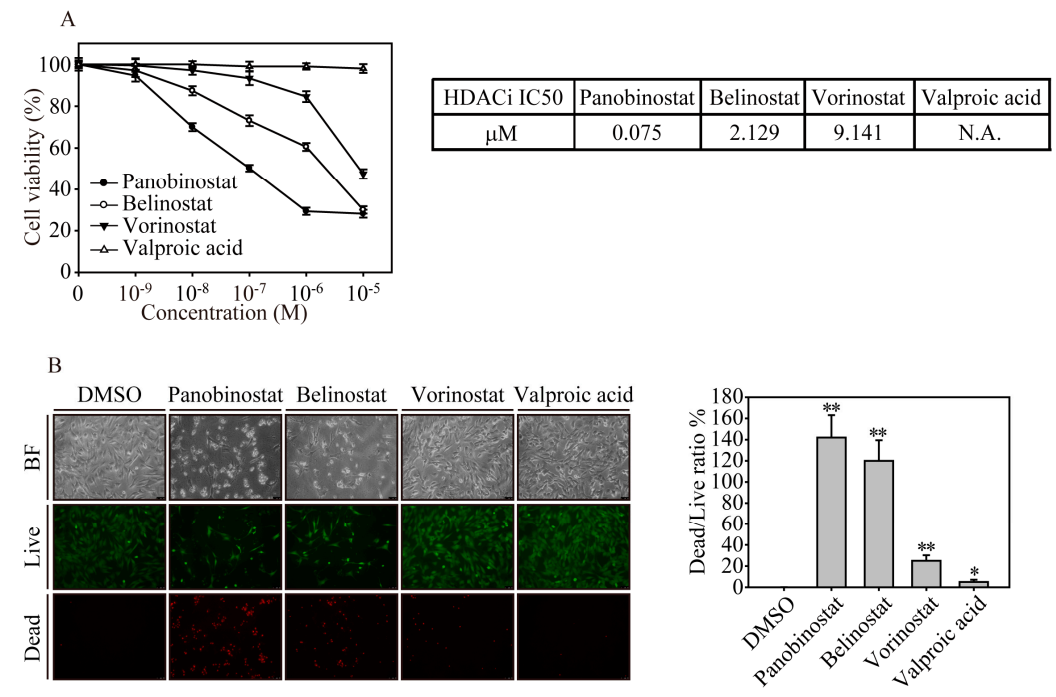

Figure 1. FDA-approved HDACi drugs significantly induced cell apoptosis in SW579 squamous-cell thyroid carcinoma (STC). (A) Cell viability of SW579 cells treated with four HDACi drugs at different concentrations $(0.001,0.01,0.1,1$ and $10 \mu \mathrm{M})$ for $24 \mathrm{~h}$ analyzed by an MTT assay. The IC50 of HDACi drugs was the drug concentration that induced a $50 \%$ inhibition of cell viability. The cell viability values are presented as the means and standard deviation. The experiment was conducted at least in triplicate. (B) Live/dead cell viability assay. The brightfield and fluorescence images of HDACi-treated SW579 cells at $1 \mu \mathrm{M}$ for $24 \mathrm{~h}$. The cells were costained with $1 \mu \mathrm{M}$ calcein-AM and $10 \mu \mathrm{M}$ PI and live/dead cells were analyzed with fluorescence microscopy. The viable cells showed green fluorescence with light emission at a wavelength of $488 \mathrm{~nm}$, whereas the dead cells showed red fluorescence in the nucleus with light emission at a wavelength of $532 \mathrm{~nm}$. The ratio of live/dead cells after HDACi treatments was plotted with bars. Scale bar represents $10 \mu \mathrm{m}$, and the magnification is $100 \times$. Data are presented as the mean and standard deviation. Data were analyzed with Student's $t$-test and all $p$-values were two-sided. $p$-values less than 0.05 are indicated with an asterisk and $p$-values less than 0.01 are indicated with two asterisks.

\subsection{HDACi Induced Histone Acetylation and Apoptosis-Related Protein Expression}

Next, we investigated the acetylation sites of the histone complex that are activated by HDACi drugs in SW579 cells (Figure 2A). According to the cell viability results, we excluded valproic acid in the following investigations due to its minor effect on SW579 cells. After 6 h of HDACi treatment, panobinostat significantly induced histone $\mathrm{H} 3$ acetylation at amino acids 9, 18 and 56, whereas histone $\mathrm{H} 4$ was acetylated at sites 8 and 16. In contrast, belinostat was the second most effective drug that induced $\mathrm{H} 3$ and $\mathrm{H} 4$ histone acetylation in SW579 cells compared to vorinostat treatment. Furthermore, we also observed that panobinostat is the only drug that significantly induced p21 protein expression and activated cell apoptosis-related proteins, such as caspase and PARP (C-caspase and C-PARP), in SW579 cells, whereas GAPDH protein expression remained unchanged. 
In a previous study, we established a bioluminescence-based live cell noninvasive apoptosis detection sensor (NIADS) system to evaluate the quantitative and kinetic characteristics of apoptotic cell death [14]. Using this assay, we were able to determine apoptotic events by simply measuring the bioluminescence activity in live cells. In this study, we used NIADS stably expressing SW579 cells (NIADS-SW579 cells), which were treated with different doses of HDACi drugs for $24 \mathrm{~h}$ and analyzed bioluminescence activity with in vivo imaging system (IVIS) (Figure 2B). The bioluminescence activity of NIADS-SW579 cells showed significant increases for the 0.1 and $1 \mu \mathrm{M}$ panobinostat and belinostat treatments compared with the DMSO control. In addition, cells treated with $1 \mu \mathrm{M}$ vorinostat had a higher bioluminescence activity than cells treated with $0.1 \mu \mathrm{M}$ vorinostat or DMSO. However, we observed that the bioluminescence activity of cells treated with $1 \mu \mathrm{M}$ panobinostat was less than that of cells treated with $0.1 \mu \mathrm{M}$ panobinostat, which may be due to a strong apoptotic event causing less cell survival in $1 \mu \mathrm{M}$ panobinostat-treated SW579 cells.
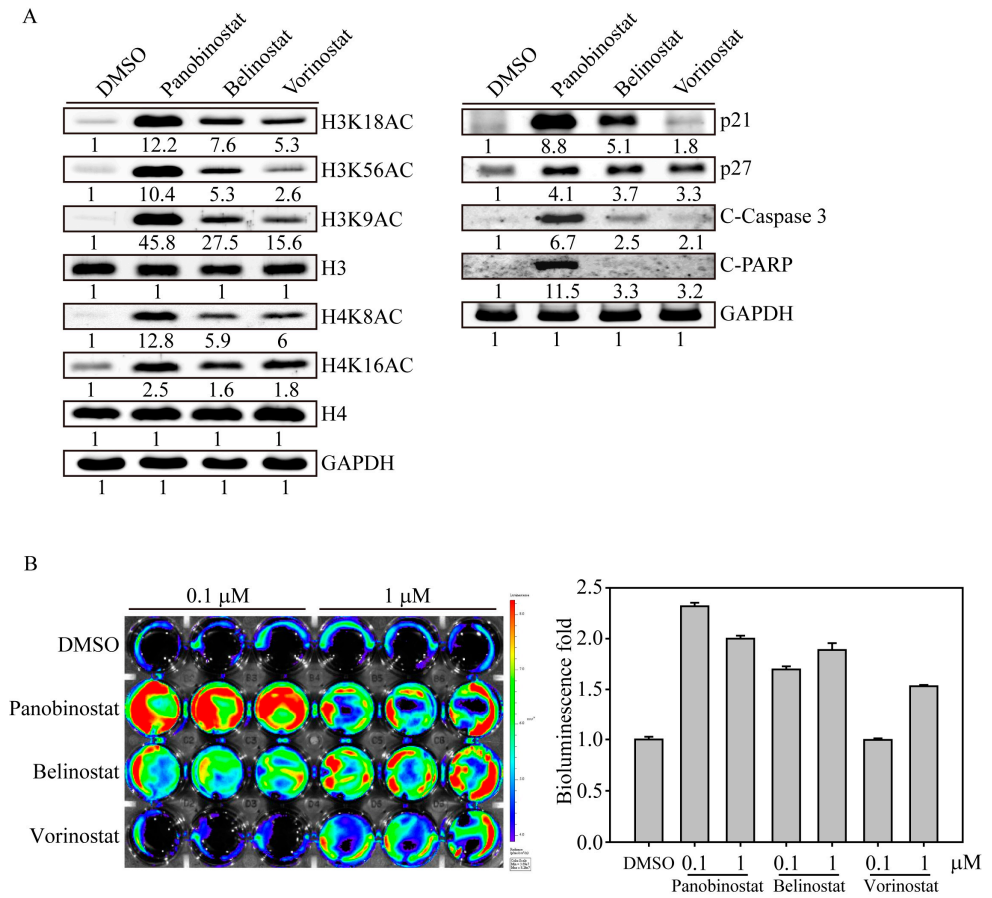

Figure 2. HDACi-induced histone acetylation and apoptosis-related protein expression. (A) HDACi drugs (panobinostat, belinostat and vorinostat) induced acetylation, cell cycle and apoptotic protein expression in SW579 cells. After $6 \mathrm{~h}$ of drug treatment, the cell lysate was collected and immunoblotted with different $\mathrm{H3}$ (H3K9AC, H3K18AC and H3K56AC) and H4 (H4K8AC and H4K16AC) histone acetylation antibodies. After SW579 cells were treated for $24 \mathrm{~h}$, the cell lysate was analyzed for cell cycle (p21 and p27) and apoptotic-related (C-caspase 3: cleaved caspase 3 and C-PARP: cleaved PARP) protein expression. Densitometric analysis of all western-blot was performed by ImageJ software. The protein expressions were normalized with their internal control such as H3, H4 or GAPDH expressions and shown as fold of control. (B) An IVIS image of HDACi-induced cell apoptosis in NIADS stably expressing SW579 (NIADS-KSW579) cells. The cells received an HDACi or DMSO in a dose-dependent manner $(0.1$ and $1 \mu \mathrm{M})$ for $24 \mathrm{~h}$ and the luciferase activity was analyzed (presented as the photon flux). Cells in a 24-well plate were exposed to luciferin at a concentration of $1.5 \mathrm{mg} / \mathrm{mL}$ and analyzed in an IVIS 200 Spectrum imaging system. The yellow and red colors indicate high photon counts of bioluminescence activity, whereas the green and blue colors present low photon counts of bioluminescence activity. The intense luciferase activity indicates apoptosis signals from NIADS.

\subsection{Panobinostat Dose-Dependently Induced Histone Acetylation and Apoptosis-Related Protein Expression in} SW576 Cells

With various concentrations of panobinostat treatment on SW579 cells for $6 \mathrm{~h}, 0.01 \mu \mathrm{M}$ panobinostat significantly induced histone H3K18 and H4K8 acetylation sites (Figure 3A), whereas 
H3K9, H3K56 and H4K16 were activated at $0.1 \mu \mathrm{M}$ of panobinostat addition. Furthermore, similar to the observations described above, panobinostat dramatically induced p21 protein expression and activated apoptosis signals in cells treated with $0.1 \mu \mathrm{M}$ for $24 \mathrm{~h}$. Next, we used flow cytometry to confirm panobinostat-induced SW579 cell apoptosis (Figure 3B). After dose-dependent panobinostat treatments, SW579 cells had a significant sub-G1 apoptotic cell accumulation at a concentration of $0.1 \mu \mathrm{M}$ with $16.5 \%$ of all cell populations, whereas 1 and $10 \mu \mathrm{M}$ panobinostat treatments induced $50.7 \%$ and $52.6 \%$ sub-G1 apoptotic cells of all cell populations, respectively. These results indicated that panobinostat, as a pan-HDACi, efficiently induced apoptosis of STC cells at very low concentrations. Additionally, panobinostat-induced cell apoptosis may potentially occur through H3K9, H3K56 and H4K16 histone acetylation regulation in SW579 cells.

A
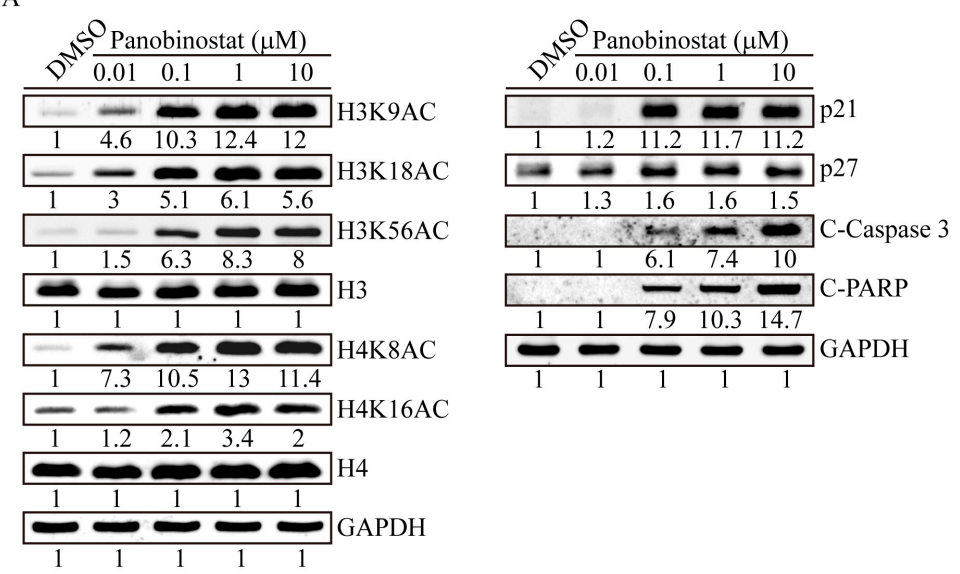

$\mathrm{B}$

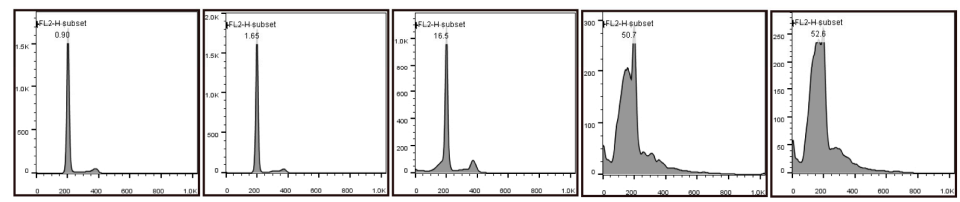

\begin{tabular}{|c|c|c|c|c|c|}
\hline Panobinostat $(\mu \mathrm{M})$ & DMSO & 0.01 & 0.1 & 1 & 10 \\
\hline Sub-G1 \% & 0.9 & 1.65 & 16.5 & 50.7 & 52.6 \\
\hline
\end{tabular}

Figure 3. Panobinostat dose-dependently induced histone acetylation and apoptosis-related protein expression in SW579 cells. (A) Cell lysates from SW579 cells treated with different concentrations (0.01 to $10 \mu \mathrm{M}$ ) of panobinostat for $6 \mathrm{~h}$ were immunoblotted with different H3 (H3K9AC, H3K18AC and H3K56AC) and H4 (H4K8AC and H4K16AC) histone acetylation antibodies. Cell lysates of SW579 cells treated with panobinostat for $24 \mathrm{~h}$ were analyzed for cell cycle (p21 and p27) and apoptotic-related (C-caspase 3: cleaved caspase 3 and C-PARP: cleaved PARP) protein expression. The H3, H4 and GAPDH immunoblots served as internal controls. Densitometric analysis of all western-blot was performed by ImageJ software. The protein expressions were normalized with their internal control such as H3, H4 or GAPDH expressions and shown as fold of control. (B) Cell cycle analysis of dose-dependent panobinostat treatments from 0.01 to $10 \mu \mathrm{M}$ on SW579 cells by flow cytometry. Apoptotic cells were determined by the sub-G1 cell population.

\subsection{HDAC1 and HDAC2 Gene Editing through the CRISPR/CAS9 System}

Next, we investigated the utility of CRISPR/Cas9 genome editing by targeting two custom-designed protospacers on HDAC1 (NM_004964.2) on chromosome 1 and the HDAC2 (NM_001527.3) locus on chromosome 6 with a lentiviral delivery system using the MIT CRISPR design website (http://crispr.mit.edu). SW579 cells transfected with scrambled (SC) lentivirus produced a wild-type $H D A C 1$ sequence (Supplementary Figure S1A,B), indicating that no gene editing occurred. In contrast, SW579 cells transfected with HDAC1 KO1 lentivirus carrying protospacer 1 (Supplementary Figure S1C) had more significant multiple gene disruptions at the predicted cleavage 
sites (red arrowhead) than HDAC1 KO2 lentivirus-transfected cells (Supplementary Figure S1D). Furthermore, TIDE analysis demonstrated that HDAC1 KO1 cells (Figure 4A) had a higher gene editing efficiency than $H D A C 1 \mathrm{KO} 2$ cells (Figure $4 \mathrm{~B}$ ), with $48 \%$ and $14.5 \%$ of the cell pool edited, respectively. The most frequent mutation in the HDAC1 $\mathrm{KO} 1 \mathrm{cell}$ pool was other mutations $(85.2 \%$, Figure $4 \mathrm{C}$ ), whereas the frequently predicted mutation in the $\mathrm{HDAC1} \mathrm{KO} 2$ cell pool was a 1-bp insertion (8.3\%, Figure 4D). Compared to HDAC1 KO2 cells, SW579 cells transduced with HDAC1 $\mathrm{KO} 1$ caused more significant gene disruptions in the targeted regions, with mutations primarily at the predicted cleavage sites (Supplementary Figure S1E,F). However, both protospacer 1- and protospacer 2-containing HDAC2 lentivirus targeted the plus strand of exon 1 on the HDAC2 gene. Sanger sequencing showed no evidence of gene editing on SC lentivirus-transduced SW579 cells (Supplementary Figure S1G,H). Compared to HDAC2 KO2 cells (Supplementary Figure S1J), HDAC2 KO1 cells (Supplementary Figure S1I) showed significant multiple gene disruptions at the predicted cleavage sites (red arrowhead). Using TIDE analysis, HDAC2 KO1 cells (Figure 4E) also showed more considerable gene editing efficiency than HDAC2 KO2 cells (Figure $4 \mathrm{~F}$ ), with $56.4 \%$ and $10.3 \%$ of the cell pool edited, respectively. The most frequent mutation in the HDAC2 KO1 cell pool was a 1-bp insertion $(29.2 \%$, Figure $4 \mathrm{G})$, whereas the frequently predicted mutation in the HDAC2 $\mathrm{KO} 2$ cell pool was a 1-bp insertion $(10.3 \%$, Figure $4 \mathrm{H})$. In addition, only HDAC2 KO1 caused significant gene disruptions in the targeted regions, whereas no gene disruptions were observed in HDAC2 KO2 SW579 cells, with mutations primarily at the predicted cleavage sites (Supplementary Figure S1K,L).

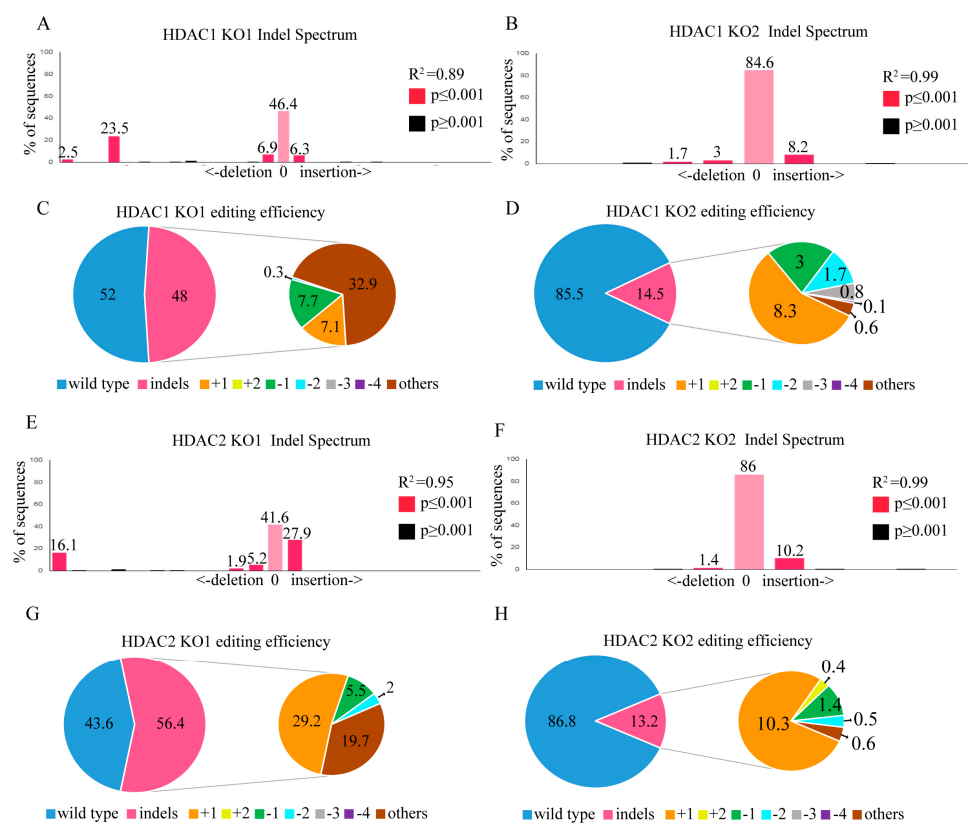

Figure 4. HDAC1 and HDAC2 gene editing of SW579 cells using the CRISPR/Cas9 system. Scrambled (SC) sgRNA and HDAC1 sgRNA were delivered to SW579 cells by lentivirus. After transduction, DNA from virus-infected cells was purified and subjected to Sanger sequencing of HDAC1 exon 2. The TIDE algorithm analysis is shown for (A) HDAC1 KO1 and (B) HDAC1 KO2 virus-transfected SW579 cells compared to SC SW579 cells. The pie charts show the percentages of indels in the HDAC1 gene edited by HDAC1 (C) KO1 and (D) KO2 lentivirus. The gene editing efficiency of the two KO cells is shown in pink, while the two most common other mutations and +1 are shown in brown and yellow colors, respectively. SC sgRNA and HDAC2 sgRNA were delivered to SW579 cells by lentivirus. After transduction, DNA from virus-infected cells was purified and subjected to Sanger sequencing of HDAC2 exon 2. The TIDE algorithm analysis is shown for (E) HDAC2 KO1 and (F) HDAC2 KO2 virus-transfected SW579 cells compared to SC SW579 cells. The pie charts show the percentages of indels in the HDAC2 gene edited by HDAC2 (G) KO1 and $(\mathbf{H}) \mathrm{KO} 2$ lentivirus. The gene editing efficiency of the two $\mathrm{KO}$ cells is shown in pink, while the two most common other mutations and +1 are shown in brown and yellow colors, respectively. 


\subsection{HDAC1 and HDAC2 Knockout Activates H3 and H4 Histone Acetylations}

Next, we evaluated HDAC1 and HDAC2 protein expression in the gene-edited SW579 cells by western blotting (Figure 5A). Similar to the abovementioned genomic results, the protein expression levels of both HDAC1 and HDAC2 virus-transfected KO1 SW579 cells were significantly decreased compared to SC virus-transfected cells. In addition, HDAC1 KO1 cells slightly gained higher HDAC2 protein expression levels, whereas $\mathrm{HDAC} 2 \mathrm{KO} 1$ cells gained higher $\mathrm{HDAC} 1$ protein expression levels in both gene-edited SW579 cells. This observation proves evidence that HDAC1 and HDAC2 have complementary effects. In the screening of histone acetylation in SW579 cells, we observed that HDAC1 KO1 cells obtained higher H3K9, H3K18, H3K56, H4K8 and H4K16 acetylation sites than HDAC2 KO1 cells. The cell arrest proteins p21 and p27 were also dramatically induced in HDAC1 cells. Next, we examined this controversial result by an MTT cell viability assay (Figure 5B). During a five-day observation, SC, HDAC1 KO1, HDAC1 KO2, HDAC2 $\mathrm{KO} 1$ and HDAC2 $\mathrm{KO} 2$ cells showed no differences in cell proliferation, confirming that the lack of HDAC1 or HDAC2 may compensate for each other by protein expression or cell signaling crosstalk.

A
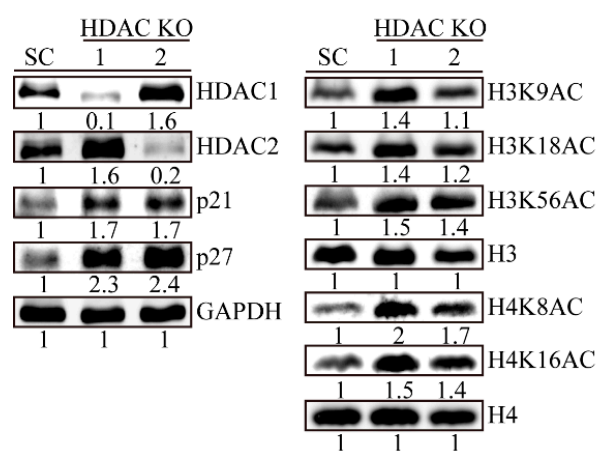

B

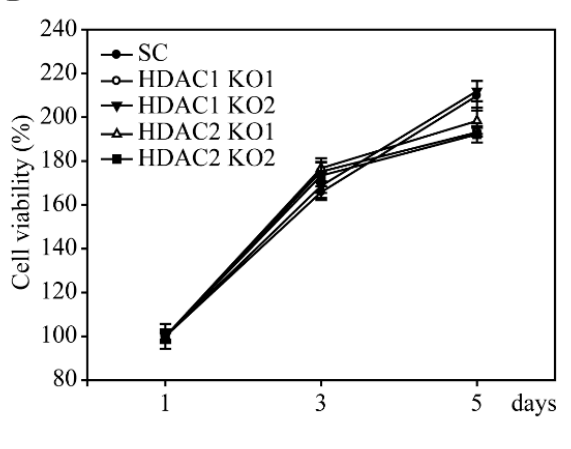

Figure 5. HDAC1 and HDAC2 KO SW579 cells induced $\mathrm{H} 3$ and $\mathrm{H} 4$ acetylation and cell arrest proteins. (A) Immunoblot analysis of gene expression in HDAC1 and HDAC2 KO SW579 cells. The cell lysates from SC, HDAC1 KO1, HDAC1 KO2, HDAC2 KO1 and HDAC2 $\mathrm{KO} 2$ cells were immunoblotted with different H3 (H3K9AC, H3K18AC and H3K56AC) and H4 (H4K8AC and H4K16AC) histone acetylation antibodies. SW579 cell lysates were also examined for cell cycle (p21 and p27) protein expression. The H3, H4 and GAPDH immunoblots served as internal controls. Densitometric analysis of all western-blot was performed by ImageJ software. The protein expressions were normalized with their internal control such as H3, H4 or GAPDH expressions and shown as fold of control. (B) MTT cell viability assay of $\mathrm{SC}, H A D C 1$ and $H D A C 2 \mathrm{KO}$ cells for 5 days. The cell viability values are presented as the means and standard deviation. The experiment was conducted at least in triplicate.

\subsection{HDAC1 and HDAC2 Double Knockout Induced Cell Apoptosis in SW579 Cells}

To investigate the compensation effect of HDAC1 and HDAC2 in maintaining cell survival on SW579 cells, we used either HDAC1 or HDAC2 KO cells that were additionally transfected with HDAC2and HDAC1-targeting lentivirus to create two individual $H D A C 1$ and $H D A C 2$ double knockout cells (HDAC1,2 KO). Using western blotting (Figure 6A), we observed that the HDAC1 protein expression level was significantly decreased in $H D A C 1 \mathrm{KO}$ and $H D A C 1,2 \mathrm{KO}$ cells, whereas the HDAC2 protein expression level was significantly decreased in $H D A C 2 \mathrm{KO}$ and $H D A C 1,2 \mathrm{KO}$ cells compared to SC SW579 cells. Interestingly, p21 and p27 cell cycle arrest-related protein expression levels were dramatically increased in $H D A C 1, H D A C 2$ and $H D A C 1,2 \mathrm{KO}$ cells. Notably, apoptosis-related proteins, such as cleaved caspase 3 and PARP, were only observed in HDAC1,2 KO cells compared to SC, $H D A C 1$ and HDAC2 cells. Using a live/dead cell assay (Figure 6B), we confirmed that two individual HDAC1,2 KO cells showed substantial apoptotic events (red), with $101 \%$ and $115 \%$ dead/live cell ratios $(p<0.01)$, whereas the cell death of HDAC1 and HDAC2 KO cells was relatively minorly affected. This result showed strong evidence that maintaining both HDAC1 and HDAC2 is essential for cell 
survival in SW579 cells. Therefore, using pan-HDACi drugs for advanced thyroid malignancy would be an effective clinical anticancer strategy in the future.

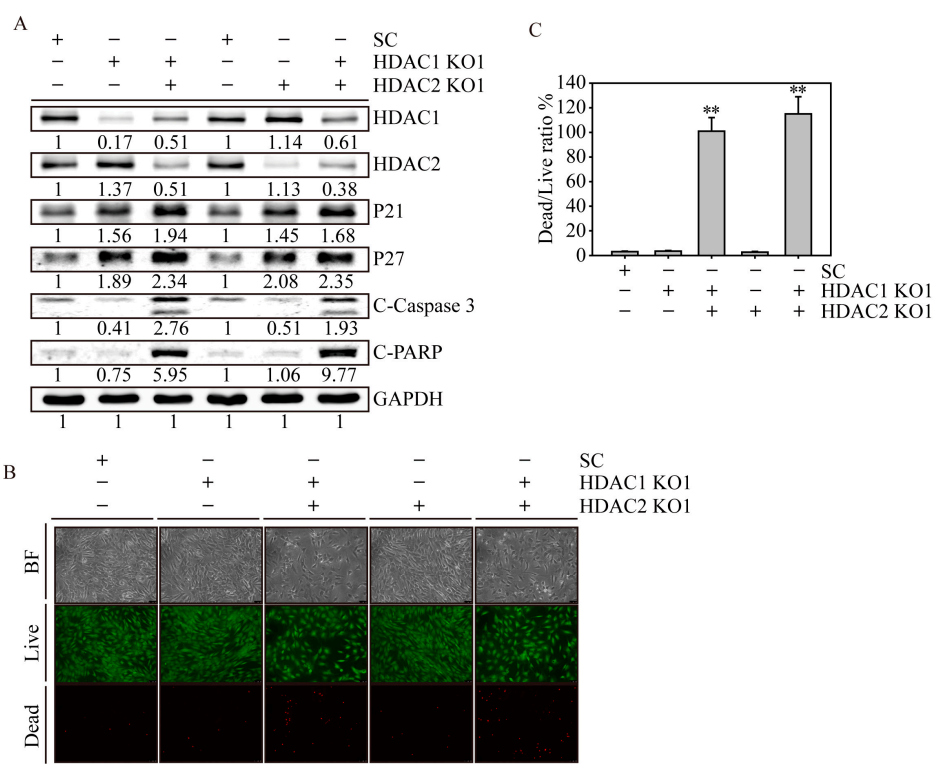

Figure 6. $H D A C 1$ and $H D A C 2$ double knockout SW579 cells induced cell cycle arrest and apoptosis. (A) Immunoblot analysis of protein expression in SC, HDAC1, HDAC2 and HDAC1,2 KO SW579 cells. The cell lysate was analyzed for HDAC1 and HDAC2 expression and cell cycle (p21 and p27) and apoptosis-related (C-Caspase 3: cleaved caspase 3 and C-PARP: cleaved PARP) protein expression. GAPDH immunoblots served as internal controls. Densitometric analysis of all western-blot was performed by ImageJ software. The protein expressions were normalized with their internal control such as $\mathrm{H} 3, \mathrm{H} 4$ or GAPDH expressions and shown as fold of control. (B) Live/dead cell viability assay of SC, HDAC1, HDAC2 and HDAC1,2 KO SW579 cells. The cells were costained with $1 \mu \mathrm{M}$ calcein-AM and $10 \mu \mathrm{M}$ PI and live/dead cells were analyzed using fluorescence microscopy. The viable cells showed green fluorescence with light emission at a wavelength of $488 \mathrm{~nm}$, whereas the dead cells showed red fluorescence in the nucleus with light emission at a wavelength of $532 \mathrm{~nm}$. The brightfield images show the morphology of gene-edited SW579 cells. Scale bar represents $10 \mu \mathrm{m}$, and the magnification is 100×. (C) The dead/live cell ratio of gene-edited SW579 cells. Data are presented as the mean and standard deviation. Data were analyzed with Student's $t$-test and all $p$-values were two-sided. $p$-values less than 0.01 are indicated with two asterisks.

\section{Discussion}

During the last two decades, thyroid cancer has increased at a higher rate than any other cancer worldwide [15], which is attributed to the genetic and environmental changes that create epigenetic modifications. Most previous studies have focused on well-differentiated thyroid cancer (WDTC), including papillary thyroid cancer (PTC) and follicular thyroid cancer (FTC), because of their prevalence and the availability of samples [16]. According to the results, the epigenetic mechanisms of histone modification and miRNA regulation seem to play an important role in thyroid tumorigenesis [17-19]. In addition, global methylation of 83 primary WDTC tumors as well as 8 samples of adjacent normal tissues identified that DNA methylation is an important mechanism that regulates signaling pathways during WDTC development, whereas etoposide-induced 2.4 (EI24) and Wilms tumor 1 (WT1) are novel prognostic markers related to recurrence-free survival [20]. Based on the evidence presented in this study, considerable epigenetic alternations, such as HDAC1 and HDAC2, in advanced thyroid cancer were investigated and more epigenetic alterations are being studied.

By increasing histone protein acetylation, HDACis lead to DNA remodeling and transcriptional activation of genes. Panobinostat is an oral pan-HDAC inhibitor that has been approved for third-line therapy of relapsed multiple myeloma [21]. The study showed that ATC cell lines, including BHT-101, 
CAL-62 and 8305C, exposed to panobinostat showed loss of cell viability, inhibition of colony formation, arrest of the cell cycle and induction of apoptosis [22]. Furthermore, animal data confirmed the cytotoxic properties of panobinostat, showing that the drug significantly reduced the expression of the Ki67 proliferation marker and therefore impaired tumor growth of ATC xenografts. In this study, the mechanisms underlying the cytotoxic effect of panobinostat on the SW579 squamous-cell thyroid carcinoma cell line included both apoptosis induction and cell cycle arrest. Apoptosis was demonstrated by the increased percentage of cells observed in the live/dead assay, sub- $\mathrm{G}_{1}$, induction of NIADS and by the activation of both PARP and caspase 3. The effect of panobinostat on cell cycle progression showed a distinctive feature; the arrest in $\mathrm{G}_{2} / \mathrm{M}$ was observed after treatment with higher doses of the drug (approximately $0.1 \mu \mathrm{M}$ ). This observation is consistent with a previous study showing that HDACis typically determine cytotoxicity at higher doses, whereas they induce $\mathrm{G}_{2} / \mathrm{M}$ arrest at lower doses [23].

The quantification of apoptosis-related cell death is an integral component of exploring cell biology and responses to cellular stress and performing high-throughput drug screens [24]. However, currently available quantitative apoptosis assays, such as the annexin V/FITC assay, require extensive sample handling and substantial labor for cell harvest and analysis. Therefore, a quantitative apoptosis assay with easy handling (the NIADS assay) was established in our previous study [14], with notable advantages, such as relatively low cell numbers, trace amounts of bioluminescence, viral infection and quick assay. These unique strengths make apoptosis detection assays suitable for high-throughput cell-based screening of drug libraries and related applications. The limitation of this assay was that higher doses of panobinostat treatment on SW579 cells resulted in lower bioluminescence signals (Figure 2B). The disadvantage was due to the strong anticancer effect of panobinostat causing less SW579 cell survival on the plate, whereas NIADS detection requires cell membranes to remain intact. To improve this assay, we aim to integrate a reporter in the current NIADS system. Therefore, the bioluminescence signals of NIADS could be normalized with internal control reporter signals. This adjustment could provide more accurate and reliable apoptosis analysis in vitro and in vivo.

A previous study demonstrated that panobinostat inhibited cell viability and colony formation abilities, whereas enhanced cell cycle arrest and apoptosis induction on three ATC cell lines (BHT-101, CAL-62 and 8305C) [22]. In animal study, a SCID xenograft model showed intraperitoneal injection of panobinostat $20 \mathrm{mg} / \mathrm{kg} /$ day of panobinostat group significantly decreased tumor growth for 2.5-fold, compared to control mice. The recent study also confirms this finding by using belinostat and panobinostat on ATC and unclassified thyroid cancer cells [25], whereas animal model showed a prominent inhibition of BHP2-7 tumor growth of the tumors occurred in the belinostat intraperitoneal injected mice $(100 \mathrm{mg} / \mathrm{kg} /$ day). To summarize, the current study once again confirms the anti-thyroid cancer ability by panobinostat or belinostat treatments, appears to be a promising therapeutic agent for the advanced cancer which is known not to respond to conventional therapy.

Previous studies have shown that knockdown of either HDAC1 or HDAC2 resulted in the sensitization of chronic lymphocytic leukemia cells to Trail-induced apoptosis [26] and a reduction in the proliferation of colon cancer cells [27]. These results indicate that the similar effects of the inactivation of either HDAC1 or HDAC2 lead to cancer cell growth suppression. In this study, we observed a compensatory mechanism for both HDAC1 and HDAC2 in SW579 cells. CRISPR/Cas9 mediated HDAC1 knockout, leading to HDAC2 protein upregulation, while HDAC2 ablation resulted in increased HDAC1 expression levels (Figure 5A). These results are consistent with other reported mouse knockout studies [28,29]. Thus, using pan-HDACi drugs to target multiple HDACs could be an effective antitumor strategy for clinical treatment of thyroid cancer and could potentially increase the survival rate of advanced thyroid cancer patients.

\section{Materials and Methods}

\subsection{Cell Culture}

The human gland epithelial squamous cell carcinoma cell line SW579 was purchased from the Bioresource Collection and Research Center (BCRC, Hsinchu, Taiwan). The cells were maintained in 
Dulbecco's modified Eagle's medium nutrient mixture F-12 (DMEM/F-12) (Gibco, Carlsbad, CA, USA). The cells were cultured with 10\% $(v / v)$ fetal bovine serum (FBS, Biological Industries, Kibbutz Beit Kaemek, Israel), 100 units $/ \mathrm{mL}$ penicillin and $100 \mathrm{mg} / \mathrm{mL}$ streptomycin and were incubated at $37^{\circ} \mathrm{C}$ with $5.0 \% \mathrm{CO}_{2}$. The medium was replaced every two days and when cells reached $80 \%$ confluence, they were passaged using $0.25 \%$ trypsin/EDTA (Gibco, CA, USA).

\subsection{MTT Cell Viability Assay}

Cell viability was analyzed using a 3-(4,5-dimethylthiazol-2-yl)-2,5-diphenyltetrazolium (MTT) assay, which is based on the reduction of yellow MTT to purple formazan by living cells [30,31]. A total of $5 \times 10^{4}$ SW579 cells were seeded in a 96 multiwell plate overnight before HDACi treatment. After $24 \mathrm{~h}$, the medium was changed to fresh medium containing $1 \mathrm{~g} / \mathrm{mL}$ MTT reagent for two $\mathrm{h}$. DMSO was added as a solvent in each well and the absorbance OD at a wavelength of $570 \mathrm{~nm}$ was obtained.

\subsection{Live/Dead Cell Assay}

SW579 cells were seeded in a 12 multiwell plate at an appropriate cell density and cultured overnight. After the indicated drug treatment, the medium was replaced with culture medium containing $1 \mu \mathrm{M}$ calcein-AM and $10 \mu \mathrm{M}$ propidium iodide (PI) for $30 \mathrm{~min}$ of incubation. The cells were analyzed by the live/dead cell assay using fluorescence microscopy. The viable cells showed green fluorescence with light emission at a wavelength of $488 \mathrm{~nm}$ and the dead cells showed red fluorescence in the nucleus with light emission at a wavelength of $532 \mathrm{~nm}$.

\subsection{Cellular Bioluminescence (IVIS) Assay}

Bioluminescence imaging was performed using an in vivo imaging system (IVIS; Xenogen) with a highly sensitive and cooled CCD camera mounted in a light-tight specimen box. SW579 cells were seeded in a 24-well multiwell plate and treated with the HDACi at concentrations of 0.1 and $1 \mu \mathrm{M}$ for $24 \mathrm{~h}$. D-Luciferin at a final concentration of $1.5 \mathrm{mg} / \mathrm{mL}$ was added to multiwell plates during imaging. The bioluminescent light from the cells was detected by the IVIS camera system and integrated, digitized and displayed. The light from each well was quantified and expressed as the total photon count using Living Image ${ }^{\circledR}$ software 4.0 (Caliper, Alameda, CA, USA).

\subsection{Flow Cytometry Analysis}

SW579 cells $\left(1 \times 10^{6}\right.$ cells / dish) were plated in a 6-cm dish and exposed to the HDACi for $24 \mathrm{~h}$. SW579 cells were collected, washed once with PBS, fixed with 75\% alcohol and analyzed with sub-G1 cell populations by flow cytometry (FACSCalibur, BD Biosciences, San Jose, CA, USA).

\subsection{Protein Extraction, Western Blotting and Antibodies}

For western blot analysis, SW579 cells were collected and washed once with ice-cold PBS, followed by the addition of radio-immunoprecipitation assay (RIPA) lysis buffer, which contained protease inhibitors. Fifty micrograms of protein from each sample was resolved by sodium dodecyl sulfate-polyacrylamide gel electrophoresis (SDS-PAGE) and transferred to a nitrocellulose membrane. The information of primary antibodies and the secondary antibodies used in this study is provided in Supplementary Table 1. All primary antibodies were used at a 1:1000 dilution with overnight hybridization, followed by a one-h incubation with a 1:4000 dilution of the secondary antibodies. Densitometric analysis of all western-blot was performed by ImageJ software (U.S. National Institutes of Health, Bethesda, MD, USA), whereas the protein expressions were normalized with its internal control such as $\mathrm{H} 3, \mathrm{H} 4$ or GAPDH expressions and shown as fold of control. 


\subsection{Lentivirus Production and Cell Transduction}

HDAC1- and HDAC2-targeting lentiviral particles and NIADS lentivirus were produced by transient transfection of Phoenix-ECO cells (CRL-3214) using TransIT ${ }^{\circledR}$-LT1 Reagent (Mirus Bio LLC, Madison, WI, USA). Guide oligonucleotides were phosphorylated, annealed and cloned into the BsmBI site of the lentiCRISPR v2 vector (Addgene, 52961, kindly provided by Feng Zhang) according to the Zhang laboratory protocol [32] (F. Zhang Laboratory, MIT, Cambridge, MA, USA). All plasmid constructs were verified by sequencing. The HDAC1, HDAC2 and NIADS plasmids were cotransfected with pMD2.G (Addgene plasmid \#12259) and psPAX2 (Addgene plasmid \#12260), which were both kindly provided by Didier Trono, EPFL, Lausanne, Switzerland. Lentiviral particles were collected at 36 and $72 \mathrm{~h}$ and then concentrated with a Lenti-X Concentrator ${ }^{\circledR}$ (Clontech, Mountain View, CA, USA). The lentiviral particles were analyzed by Q-PCR [31]. A total of $1 \times 10^{6}$ SW579 cells were plated in a 6-cm dish and treated with lentivirus at an MOI of 5. Two days after transfection, the medium was replaced with medium containing $2.5 \mathrm{mg} / \mathrm{mL}$ puromycin for two days. Lentivirus-transfected cells were recovered two days before the experiments.

\subsection{Sequencing of Single Guide RNA ( $\mathrm{g} g$ RNA) Target Sites}

Genomic DNA was extracted and PCR was used to amplify the HDAC1 and HDAC2 gene region using exon 2 DNA primers, which are listed in Supplementary Table 2, whereas sgRNA sequence were underlined. The PCR products were purified using a PCR clean-up purification kit and were sequenced by the Sanger method using forward PCR primers. The editing efficiency of the sgRNAs and the potential induced mutations were assessed using Tracking of Indels by Decomposition (TIDE) software (https: / / tide-calculator.nki.nl; Netherlands Cancer Institute, Amsterdam, The Netherlands), which only required two Sanger sequencing runs from wild-type and mutated cells.

\subsection{Statistical Methods}

All data are presented as the mean \pm SD. Student's $t$-test analysis was performed for the pairwise samples. All bar graphs were plotted using SigmaPlot graphing software (version 10.0, Systat software Inc, Chicago, IL, USA). The statistical analyses were performed using Statistical Package for the Social Sciences v.13 software (SPSS, Chicago, IL, USA). All statistical tests were two-sided. A $p$-value of 0.05 or less was considered to indicate statistical significance. $p$-values less than 0.05 are indicated with an asterisk and $p$-values less than 0.01 are indicated with two asterisks.

\section{Conclusions}

The 5-year survival rate of thyroid cancer is greater than $90 \%$. However, the most aggressive and malignant thyroid cancer types, such as anaplastic thyroid carcinoma (ATC) and squamous thyroid carcinoma (STC), both have a very poor prognosis. In the current study, we observed that HDAC1 and HDAC2 have compensatory effects, which play important roles in maintaining cell survival in aggressive STC SW579 cells. By introducing sgRNAs of both HDAC1 and HDAC2 to edit these genes, cell cycle arrest and apoptotic events of SW579 cells were significantly enhanced. In addition, we observed that pan-HDACi drug treatment using panobinostat resulted in a substantially greater anticancer effect on SW579 cells compared to other HDACi drugs. This could also be effective in the clinical treatment of aggressive thyroid cancer.

Supplementary Materials: Supplementary materials can be found at http:/ / www.mdpi.com/1422-0067/20/2/ 454/s1.

Author Contributions: C.-L.L. and C.-H.L. designed the entire study. C.-Y.L. designed the HDAC knockout sequence. K.-W.H. and C.-H.L. designed the virus production and infection procedures. B.-M.S. performed western blotting in this study. C.-L.L., M.-L.T., W.-S.H., W.-M.C. and L.-C.H. participated in the clinical discussion. C.-L.L., L.-C.H. and C.-H.L. conceived the study and supervised the project. All authors read and approved the final manuscript. 
Funding: This study was supported by Cathay General Hospital by the grants CGH-MR-A10721 and CGH-MR-A10629 for L.-C.H., the China Medical University by the grant CMU107-N-07 for K.-W.H., the Ministry of Science and Technology grant 102-2320-B-038-039-MY3 for C.-H.L. and the Health and Welfare Surcharge of Tobacco Products grant MOHW107-TDU-B-212-114014 for C.-H.L. Meanwhile, this study was financially supported by the "TMU Research Center of Cancer Translational Medicine" from The Featured Areas Research Center Program within the framework of the Higher Education Sprout Project by the Ministry of Education (MOE) in Taiwan. None of the funding bodies were involved in the design of this study, the collection, analysis and interpretation of the data or the preparation of the manuscript.

Acknowledgments: C.-Y.L. thanks the Japan Society for the Promotion of Science (JSPS) International Research Fellowship (ID: P17353) for support.

Conflicts of Interest: The authors declare no conflict of interest.

\section{Abbreviations}

$\begin{array}{ll}\text { STC } & \text { Squamous thyroid carcinoma } \\ \text { HDACi } & \text { Histone deacetylase inhibitor } \\ \text { NIADS } & \text { Noninvasive apoptosis detection sensor } \\ \text { KO } & \text { Knockout } \\ \text { CRISPR/Cas9 } & \text { Clustered regularly interspaced short palindromic repeat } \\ \text { IVIS } & \text { in vivo imaging system }\end{array}$

\section{References}

1. Koyama, S.; Fujiwara, K.; Nosaka, K.; Fukuhara, T.; Morisaki, T.; Miyake, N.; Kitano, H.; Takeuchi, H. Immunohistochemical Features of Primary Pure Squamous Cell Carcinoma in the Thyroid: An Autopsy Case. Case Rep. Oncol. 2018, 11, 418-424. [CrossRef]

2. Keutgen, X.M.; Sadowski, S.M.; Kebebew, E. Management of anaplastic thyroid cancer. Gland Surg. 2015, 4, 44-51. [PubMed]

3. Struller, F.; Senne, M.; Falch, C.; Kirschniak, A.; Konigsrainer, A.; Muller, S. Primary squamous cell carcinoma of the thyroid: Case report and systematic review of the literature. Int. J. Surg. Case Rep. 2017, 37, 36-40. [CrossRef] [PubMed]

4. Kebebew, E. Anaplastic thyroid cancer: Rare, fatal and neglected. Surgery 2012, 152, 1088-1089. [CrossRef] [PubMed]

5. Liu, Z.; Liu, G.; Liu, X.; Li, S. The effects of hyperoside on apoptosis and the expression of Fas/FasL and survivin in SW579 human thyroid squamous cell carcinoma cell line. Oncol. Lett. 2017, 14, 2310-2314. [CrossRef]

6. Saini, S.; Tulla, K.; Maker, A.V.; Burman, K.D.; Prabhakar, B.S. Therapeutic advances in anaplastic thyroid cancer: A current perspective. Mol. Cancer 2018, 17, 154. [CrossRef] [PubMed]

7. Bae, J.M. Researches of Epigenetic Epidemiology for Infections and Radiation as Carcinogen. J. Prev. Med. Public Health 2018, 51, 169-172. [CrossRef] [PubMed]

8. Chmielik, E.; Rusinek, D.; Oczko-Wojciechowska, M.; Jarzab, M.; Krajewska, J.; Czarniecka, A.; Jarzab, B. Heterogeneity of Thyroid Cancer. Pathobiology 2018, 85, 117-129. [CrossRef] [PubMed]

9. Khatami, F.; Tavangar, S.M. Genetic and Epigenetic of Medullary Thyroid Cancer. Iran Biomed. J. 2018, 22, 142-150.

10. Sasanakietkul, T.; Murtha, T.D.; Javid, M.; Korah, R.; Carling, T. Epigenetic modifications in poorly differentiated and anaplastic thyroid cancer. Mol. Cell Endocrinol. 2018, 469, 23-37. [CrossRef]

11. Zhang, Z.; Liu, D.; Murugan, A.K.; Liu, Z.; Xing, M. Histone deacetylation of NIS promoter underlies BRAF V600E-promoted NIS silencing in thyroid cancer. Endocr. Relat. Cancer 2014, 21, 161-173. [CrossRef] [PubMed]

12. Pugliese, M.; Fortunati, N.; Germano, A.; Asioli, S.; Marano, F.; Palestini, N.; Frairia, R.; Boccuzzi, G.; Catalano, M.G. Histone deacetylase inhibition affects sodium iodide symporter expression and induces 131I cytotoxicity in anaplastic thyroid cancer cells. Thyroid 2013, 23, 838-846. [CrossRef] [PubMed]

13. Catalano, M.G.; Poli, R.; Pugliese, M.; Fortunati, N.; Boccuzzi, G. Emerging molecular therapies of advanced thyroid cancer. Mol. Aspects Med. 2010, 31, 215-226. [CrossRef] [PubMed] 
14. Hsu, K.W.; Huang, C.Y.; Tam, K.W.; Lin, C.Y.; Huang, L.C.; Lin, C.L.; Hsieh, W.S.; Chi, W.M.; Chang, Y.J.; Wei, P.L.; et al. The Application of Non-Invasive Apoptosis Detection Sensor (NIADS) on Histone Deacetylation Inhibitor (HDACi)-Induced Breast Cancer Cell Death. Int. J. Mol. Sci. 2018, 19, 452. [CrossRef] [PubMed]

15. Liu, Y.; Su, L.; Xiao, H. Review of Factors Related to the Thyroid Cancer Epidemic. Int. J. Endocrinol. 2017, 2017, 5308635. [CrossRef] [PubMed]

16. Zarkesh, M.; Zadeh-Vakili, A.; Azizi, F.; Foroughi, F.; Akhavan, M.M.; Hedayati, M. Altered Epigenetic Mechanisms in Thyroid Cancer Subtypes. Mol. Diagn. Ther. 2018, 22, 41-56. [CrossRef] [PubMed]

17. Stephen, J.K.; Chitale, D.; Narra, V.; Chen, K.M.; Sawhney, R.; Worsham, M.J. DNA methylation in thyroid tumorigenesis. Cancers 2011, 3, 1732-1743. [CrossRef]

18. Zhu, X.; Cheng, S.Y. Epigenetic Modifications: Novel Therapeutic Approach for Thyroid Cancer. Endocrinol. Metab. 2017, 32, 326-331. [CrossRef]

19. Catalano, M.G.; Fortunati, N.; Boccuzzi, G. Epigenetics modifications and therapeutic prospects in human thyroid cancer. Front. Endocrinol. 2012, 3, 40. [CrossRef]

20. Mancikova, V.; Buj, R.; Castelblanco, E.; Inglada-Perez, L.; Diez, A.; de Cubas, A.A.; Curras-Freixes, M.; Maravall, F.X.; Mauricio, D.; Matias-Guiu, X.; et al. DNA methylation profiling of well-differentiated thyroid cancer uncovers markers of recurrence free survival. Int. J. Cancer 2014, 135, 598-610. [CrossRef]

21. Yee, A.J.; Raje, N.S. Panobinostat and Multiple Myeloma in 2018. Oncologist 2018, 23, 516-517. [CrossRef] [PubMed]

22. Catalano, M.G.; Pugliese, M.; Gargantini, E.; Grange, C.; Bussolati, B.; Asioli, S.; Bosco, O.; Poli, R.; Compagnone, A.; Bandino, A.; et al. Cytotoxic activity of the histone deacetylase inhibitor panobinostat (LBH589) in anaplastic thyroid cancer in vitro and in vivo. Int. J. Cancer 2012, 130, 694-704. [CrossRef] [PubMed]

23. Johnstone, R.W.; Licht, J.D. Histone deacetylase inhibitors in cancer therapy: Is transcription the primary target? Cancer Cell 2003, 4, 13-18. [CrossRef]

24. Gelles, J.D.; Chipuk, J.E. Robust high-throughput kinetic analysis of apoptosis with real-time high-content live-cell imaging. Cell Death Dis. 2016, 7, e2493. [CrossRef] [PubMed]

25. Chan, D.; Zheng, Y.; Tyner, J.W.; Chng, W.J.; Chien, W.W.; Gery, S.; Leong, G.; Braunstein, G.D.; Koeffler, H.P. Belinostat and panobinostat (HDACI): In vitro and in vivo studies in thyroid cancer. J. Cancer Res. Clin. Oncol. 2013, 139, 1507-1514. [CrossRef]

26. Inoue, S.; Mai, A.; Dyer, M.J.; Cohen, G.M. Inhibition of histone deacetylase class I but not class II is critical for the sensitization of leukemic cells to tumor necrosis factor-related apoptosis-inducing ligand-induced apoptosis. Cancer Res. 2006, 66, 6785-6792. [CrossRef]

27. Weichert, W.; Roske, A.; Niesporek, S.; Noske, A.; Buckendahl, A.C.; Dietel, M.; Gekeler, V.; Boehm, M.; Beckers, T.; Denkert, C. Class I histone deacetylase expression has independent prognostic impact in human colorectal cancer: Specific role of class I histone deacetylases in vitro and in vivo. Clin. Cancer Res. 2008, 14, 1669-1677. [CrossRef]

28. Yamaguchi, T.; Cubizolles, F.; Zhang, Y.; Reichert, N.; Kohler, H.; Seiser, C.; Matthias, P. Histone deacetylases 1 and 2 act in concert to promote the G1-to-S progression. Genes Dev. 2010, 24, 455-469. [CrossRef]

29. Wilting, R.H.; Yanover, E.; Heideman, M.R.; Jacobs, H.; Horner, J.; van der Torre, J.; DePinho, R.A.; Dannenberg, J.H. Overlapping functions of Hdac1 and Hdac2 in cell cycle regulation and haematopoiesis. EMBO J. 2010, 29, 2586-2597. [CrossRef]

30. Lee, C.H.; Huang, C.S.; Chen, C.S.; Tu, S.H.; Wang, Y.J.; Chang, Y.J.; Tam, K.W.; Wei, P.L.; Cheng, T.C.; $\mathrm{Chu}$, J.S.; et al. Overexpression and activation of the alpha9-nicotinic receptor during tumorigenesis in human breast epithelial cells. J. Natl. Cancer Inst. 2010, 102, 1322-1335. [CrossRef]

31. Tu, S.H.; Hsieh, Y.C.; Huang, L.C.; Lin, C.Y.; Hsu, K.W.; Hsieh, W.S.; Chi, W.M.; Lee, C.H. A rapid and quantitative method to detect human circulating tumor cells in a preclinical animal model. BMC Cancer 2017, 17, 440. [CrossRef] [PubMed]

32. Shalem, O.; Sanjana, N.E.; Hartenian, E.; Shi, X.; Scott, D.A.; Mikkelsen, T.S.; Heckl, D.; Ebert, B.L.; Root, D.E.; Doench, J.G.; et al. Genome-scale CRISPR-Cas9 knockout screening in human cells. Science 2014, 343, 84-87. [CrossRef] [PubMed]

(C) 2019 by the authors. Licensee MDPI, Basel, Switzerland. This article is an open access article distributed under the terms and conditions of the Creative Commons Attribution (CC BY) license (http://creativecommons.org/licenses/by/4.0/). 